\title{
A population-based analysis of invasive fungal disease in haematology-oncology patients using data linkage of state-wide registries and administrative databases: 2005 - 2016
}

Jake C. Valentine ${ }^{1,6^{*}}$ (D) C. Orla Morrissey ${ }^{1,3}$, Mark A. Tacey ${ }^{2}$, Danny Liew ${ }^{2}$, Sushrut Patil ${ }^{3}$, Anton Y. Peleg ${ }^{1,4}$ and Michelle R. Ananda-Rajah ${ }^{1,5}$

\begin{abstract}
Background: Little is known about the morbidity and mortality of invasive fungal disease (IFD) at a population level. The aim of this study was to determine the incidence, trends and outcomes of IFD in all haematology-oncology patients by linking Victorian hospital data to state-based registries.

Methods: Episodes of IFD complicating adult haematological malignancy (HM) and haematopoietic stem cell transplantation (HSCT) patients admitted to Victorian hospitals from $1^{\text {st }}$ July 2005 to $30^{\text {th }}$ June 2016 were extracted from the Victorian Admitted Episodes Dataset and linked to the date of HM diagnosis from the Victorian Cancer Registry and mortality from the Victorian Death Index. Descriptive analyses and regression modelling were used.

Results: There were 619,702 inpatient-episodes among 32,815 HM and 1,765 HSCT-patients. IFD occurring twelvemonths from HM-diagnosis was detected in 669 (2.04\%) HM-patients and 111 (6.29\%) HSCT-recipients, respectively. Median time to IFD-diagnosis was 3, 5, 15 and 22 months in acute myeloid leukaemia, acute lymphoblastic leukaemia, Hodgkin lymphoma and multiple myeloma, respectively. Median survival from IFD-diagnosis was 7,7 and 3 months for invasive aspergillosis, invasive candidiasis and mucormycosis, respectively. From 2005-2016, IFD incidence decreased $0.28 \%$ per 1,000 bed-days. Fungal incidence coincided with spring peaks on time-series analysis.

Conclusions: Data linkage is an efficient means of evaluating the epidemiology of a rare disease, however the burden of IFD is likely underestimated, arguing for better quality hospital level surveillance data to improve management strategies.
\end{abstract}

Keywords: Invasive fungal disease, haematological malignancy, haematopoietic stem cell transplantation, data linkage, epidemiology

\footnotetext{
* Correspondence: jvalentine@student.unimelb.edu.au

${ }^{1}$ Department of Infectious Diseases, Alfred Health and Central Clinical School,

Monash University, Melbourne, Victoria, Australia

${ }^{6}$ Cancer Research Division, Level 13, Peter MacCallum Cancer Centre,

Victorian Comprehensive Cancer Centre, 305 Grattan Street, Melbourne,

Victoria 3000, Australia

Full list of author information is available at the end of the article
}

(c) The Author(s). 2019 Open Access This article is distributed under the terms of the Creative Commons Attribution 4.0 International License (http://creativecommons.org/licenses/by/4.0/), which permits unrestricted use, distribution, and reproduction in any medium, provided you give appropriate credit to the original author(s) and the source, provide a link to the Creative Commons license, and indicate if changes were made. The Creative Commons Public Domain Dedication waiver (http://creativecommons.org/publicdomain/zero/1.0/) applies to the data made available in this article, unless otherwise stated. 


\section{Background}

Invasive fungal disease (IFD) represents a significant challenge in the management of patients with haematological malignancies (HM) undergoing cytotoxic chemotherapy and/or haematopoietic stem cell transplantation (HSCT) [1]. IFD is associated with a high mortality ranging from $29-90 \%[1,2]$ and may affect long-term leukaemia outcomes by delaying or modifying curative chemotherapy or HSCT [3]. Few studies have evaluated the value of data linkage for IFD surveillance [4] and none have focused on the disease burden of these infections at a population-level in Victoria, Australia.

Administrative datasets are an efficient source of epidemiological data [5], yet their utility for IFD surveillance in Australia has not been well studied. The only population-based analysis of IFD in Australia used hospital discharge-coded data from 1995 to 1999 and showed that invasive candidiasis (IC) was more common than invasive aspergillosis (IA) representing $0.36 \%$ and $0.03 \%$ of all acute hospital discharges, respectively, and were associated with mortality rates between $8-26 \%$ for both IC and IA [4]. Importantly, these data predated the introduction of broad-spectrum triazole antifungal drugs that have resulted in a shift in fungal epidemiology to filamentous moulds [6] and it excluded the second most populous state in Australia, namely Victoria, with a population of 6.39 million residents [7]. The availability of state-based datasets has afforded an opportunity to revisit IFD disease burden and trends among haematology patients capturing the era of potent mould-active antifungal therapies and improvements in supportive care in cancer [8].

In this study, we linked existing population-based datasets and state registry data to characterise the epidemiology of IFD among the HM and HSCT populations across Victoria. The Victorian Admitted Episodes Dataset (VAED) is Australia's largest hospital morbidity database and comprises demographic, administrative and clinical information coded according to the International Statistical Classification of Diseases and Related Health Problems, Tenth Revision, Australian Modification (ICD-10-AM) associated with every hospitalisation in Victorian public and private hospitals [9]. The Victorian Cancer Registry (VCR) has recorded all cancer diagnoses from 1982 with the exception of basal and squamous cell carcinomas of the skin in Victorian residents [10], but is only available for haematological malignancies from $1^{\text {st }}$ January 2008 to the $31^{\text {st }}$ December 2014. Overall, in-hospital and out-of-hospital mortality was evaluated with linkage to the Victorian Death Index (VDI), thus allowing comparisons of survival in patients with and without IFD. We performed data linkage between the VAED, VCR and VDI to characterise the epidemiology of IFD at a population-level over a decade in order to evaluate trends, risk-factors and to identify patient groups at high-risk for IFD.

\section{Methods}

\section{Study design and setting}

This was an observational, retrospective, longitudinal study of adult patients ( $\geq 16$-years) diagnosed with a HM and/or post-allogeneic- (allo) or autologous (auto)-HSCT across Victorian public and private hospitals. All reporting parameters are consistent with the STrengthening the Reporting of OBservational studies in Epidemiology (STROBE) Statement [11] (Additional file 1).

\section{Data sources, linkage and clinical definitions}

VAED data were linked to the dates of death from the VDI between the $1^{\text {st }}$ July 2005 and the $30^{\text {th }}$ June 2016. The datasets were linked by the Victorian Data Linkages Unit (VDLU) using probabilistic and stepwise deterministic linkage. A linkage map based on encrypted statistical linkage keys for every record across each dataset was assigned to differentiate individual patients as well as reports of multiple episode-of-care for any given patient (Fig. 1).

All patients diagnosed with a HM diagnostic- or HSCT procedural code were included. An episode-of-care was defined as a hospitalisation. After linkage of the VAED with the VCR, index hospitalisation for a HM was defined as the first appearance in an episode of an ICD-10-AM code denoting a HM (Additional file 2) appearing soon after the date of HM diagnosis as recorded in the VCR (e.g. C92, acute myeloid leukaemia (AML) appearing after the date of diagnosis from the VCR). For hospitalisations outside the dates of the VCR, i.e. before $1^{\text {st }}$ January 2008 or after $31^{\text {st }}$ December 2014, the first episode-of-care recorded in the VAED was assumed to be the index hospitalisation. A HSCT-recipient was defined by the first procedural ICD-10-AM code denoting a HSCT with the corresponding date in month and year format in the VAED.

We assessed IFD incidence as the first appearance of an ICD-10-AM code for IFD in the twelve-months from index hospitalisation for a HM-patient and twelve-months post-transplantation among the HSCT-cohort. Exclusion criteria included paediatric patients ( $<16$-years) and HMor HSCT-patients diagnosed with endemic or superficial fungal infections (refer to Additional file 3 for excluded codes). Cases were defined as HM or HSCT patients who had an IFD-code assigned in the first twelve-months post HM-diagnosis or HSCT and controls were defined as patients with no IFD diagnostic code. Duplicate IFD codes occurring in the same twelve-month time period were treated as the same IFD. No censoring was used when investigating the median time to IFD-onset among the HM- and HSCT-cohorts. Hospitalisation for induction chemotherapy was defined as an episodeof-care where an ICD-10-AM procedural code for 


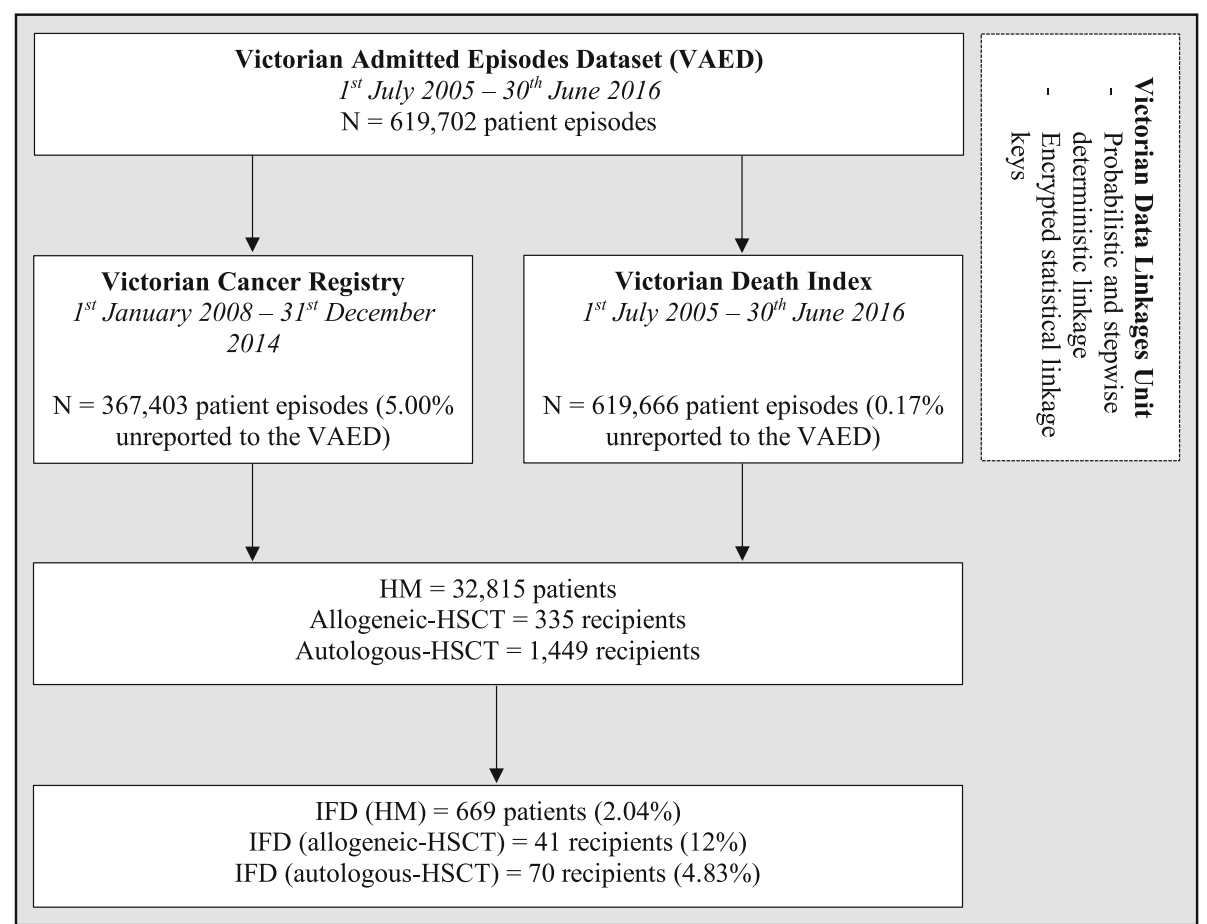

Fig. 1 Overview of the probabilistic and stepwise deterministic linkage across administrative datasets held by the State Government of Victoria. IFD, invasive fungal disease; HM, haematological malignancy; HSCT, haematopoietic stem cell transplantation; VAED, Victorian Admitted Episodes Dataset

chemotherapy first appeared either during or after the index hospitalisation.

\section{Statistical analyses}

Chi-squared $\left(\chi^{2}\right)$ and Wilcoxon rank-sum tests were used to compare the statistical significance between two categorical and non-parametric continuous covariates, respectively. A multivariable logistic regression was used to identify risk-factors for IFD since induction chemotherapy. Variables with a $\mathrm{p}<0.20$ on univariate- and $\mathrm{p}<0.05$ on multivariate analysis were included in a manual stepwise backward elimination process. To quantify the percent risk of IFD twelve-months from induction chemotherapy, a sigmoid function that uses marginal standardisation and prediction at the means described elsewhere was applied [12, 13]. A receiver operating curve and its C-statistic (Additional file 4) examined the model's discrimination power and a Hosmer-Lemeshow $\chi^{2}$ test assessed the model's calibration power.

Kaplan-Meier analysis and log-rank test were used to compare survival (in months) among HM-patients with and without IFD from the date of HM- and IFD-diagnosis, respectively. A time-series analysis of IFD incidence risk-adjusted for bed-day occupancy was developed. A two-sided p-value $<0.05$ was considered statistically significant. All statistical analyses were undertaken using
Stata/SE v14.2 software (StataCorp ${ }^{\circ}$ LLC, College Station, Texas, U.S.A.).

\section{Ethics}

Written ethics approval was granted by the Alfred Health Human Research Ethics Committee (project number: 93/17).

\section{Results}

\section{Patient characteristics}

A total of 32,815 HM-patients were identified from 619,702 hospitalisations recorded in the VAED from $1^{\text {st }}$ July 2005 to $30^{\text {th }}$ June 2016 . IFD occurred in $669(2.04 \%)$ patients within twelve-months following HM-diagnosis. Among 1,765 allo- or auto-HSCT-recipients, 111 $(6.29 \%)$ were diagnosed with an IFD twelve-months post-transplantation (Table 1).

Incidence of invasive fungal disease and clinical outcomes The distribution of IFD is presented in Table 2. The IFD incidence rate was highest in the allo-HSCT subgroup $(\mathrm{N}=48 ; 12 \%)$, followed by acute lymphoblastic leukaemia (ALL) $\quad(\mathrm{N}=75 ; 11 \%), \quad$ AML $\quad(\mathrm{N}=249 ; 9.42 \%)$, autoHSCT-recipients $(\mathrm{N}=70 ; 4.83 \%)$ and aplastic anaemia $(\mathrm{N}=7,121 ; 1.42 \%)$. Mould diseases constituted $61 \%$ of all IFD-cases compared to $39 \%$ for yeasts (Fig. 2). IA was the 
Table 1 Demographic and Clinical Characteristics of Haematological Malignancy Patients and Haematopoietic Stem Cell Transplantation Recipients, 2005 - 2016

\begin{tabular}{|c|c|c|c|c|c|c|}
\hline \multirow[t]{2}{*}{ Characteristic } & \multicolumn{3}{|c|}{ Haematological malignancy patients } & \multicolumn{3}{|c|}{ Haematopoietic stem cell transplantation recipients } \\
\hline & $\begin{array}{l}\text { Invasive fungal disease } \\
\text { cases, } \mathrm{N}=669(\%)\end{array}$ & $\begin{array}{l}\text { Invasive fungal disease } \\
\text { controls, } \mathrm{N}=32,146(\%)\end{array}$ & $\overline{p \text {-value }}$ & $\begin{array}{l}\text { Invasive fungal disease } \\
\text { cases, } \mathrm{N}=111(\%)\end{array}$ & $\begin{array}{l}\text { Invasive fungal disease } \\
\text { controls, } N=1,654(\%)\end{array}$ & $\overline{p \text {-value }}$ \\
\hline Overall crude incidence & $669 / 32,815=2.04 \%$ & $32,146 / 32,815=98 \%$ & $<0.001$ & $111 / 1,765=6.29 \%$ & $1,654 / 1,765=94 \%$ & $<0.001$ \\
\hline Age (years: median [IQR]) & $63[62-72]$ & $71[58-81]$ & $<0.001$ & $54[41-64]$ & $58[49-65]$ & 0.768 \\
\hline $15-25$ & $38(5.7)$ & $899(2.8)$ & $<0.001$ & $12(11)$ & $75(4.5)$ & 0.011 \\
\hline $26-55$ & $187(28)$ & $6,407(20)$ & & $51(46)$ & $714(51)$ & \\
\hline $56-70$ & $189(29)$ & $7,095(22)$ & & $46(41)$ & $840(51)$ & \\
\hline $71+$ & $179(27)$ & $15,042(47)$ & & $2(1.8)$ & $25(14)$ & \\
\hline \multicolumn{7}{|l|}{ Gender } \\
\hline Male & $406(60)$ & $18,364(57)$ & 0.065 & $63(57)$ & $1,030(62)$ & 0.247 \\
\hline \multicolumn{7}{|l|}{ Presence of neutropenia } \\
\hline & $549(82)$ & $6,735(21)$ & $<0.001$ & $107(96)$ & $1,478(89)$ & 0.018 \\
\hline \multicolumn{7}{|l|}{$\begin{array}{l}\text { Inpatient length of stay } \\
\text { (LOS; days) }\end{array}$} \\
\hline $\begin{array}{l}\text { Median [IQR] hospital } \\
\text { LOS }\end{array}$ & $21[8-36]$ & $1[1-1]$ & $<0.001$ & $32[25-48]$ & $19[16-24]$ & $<0.001$ \\
\hline \multicolumn{7}{|l|}{ Intensive Care Unit } \\
\hline ICU admissions & $37(5.5)$ & $585(1.8)$ & $<0.001$ & $0(0)$ & $4(0.2)$ & 1.000 \\
\hline Median [IQR] ICU LOS (days) & $6.1[2.8-16]$ & $2.5[1.1-5.3]$ & $<0.001$ & $0(0)$ & $4.0[1.9-10]$ & - \\
\hline $\begin{array}{l}\text { Mechanical ventilation } \\
\text { (yes) }\end{array}$ & $29(4.3)$ & $286(0.9)$ & $<0.001$ & $0(0)$ & $3(0.2)$ & 1.000 \\
\hline $\begin{array}{l}\text { Median [IQR] ICU mechanical } \\
\text { ventilation (days) }\end{array}$ & $6.8[2.7-17]$ & $2.0[0.6-6.0]$ & $<0.001$ & - & $180[82-280]$ & \\
\hline \multicolumn{7}{|l|}{ Haemodialysis } \\
\hline & $68(10)$ & $478(1.5)$ & $<0.001$ & $10(9.0)$ & $62(3.8)$ & 0.007 \\
\hline \multicolumn{7}{|l|}{ Overall mortality } \\
\hline One-month & $43(6.4)$ & $1,983(6.2)$ & 0.783 & $0(0)$ & $6(0.4)$ & 1.000 \\
\hline Three-month & $110(16)$ & $3,223(10)$ & $<0.001$ & $0(0)$ & $9(0.54)$ & 1.000 \\
\hline Six-month & $167(25)$ & 4,353 (14) & $<0.001$ & $4(3.60)$ & $29(1.8)$ & 0.149 \\
\hline Twelve-month & $290(43)$ & $6,004(19)$ & $<0.001$ & $20(18)$ & $108(6.5)$ & $<0.001$ \\
\hline \multicolumn{7}{|l|}{ Hospital region } \\
\hline Metropolitan Victoria & $591(88)$ & $25,077(78)$ & $<0.001$ & $102(92)$ & $1,388(84)$ & 0.025 \\
\hline Regional Victoria & $78(12)$ & $7,069(22)$ & & $9(8.11)$ & $266(16)$ & \\
\hline \multicolumn{7}{|l|}{$\begin{array}{l}\text { Charlson Comorbidity } \\
\text { Index score } \geq 4\end{array}$} \\
\hline & $192(27)$ & $3,808(12)$ & $<0.001$ & $27(24)$ & $157(9.5)$ & $<0.001$ \\
\hline
\end{tabular}

Abbreviations: ICU intensive care unit, IQR inter-quartile range, LOS length of stay

most common mould disease occurring in 31 (5.07\%) allo-HSCT-recipients and 29 (4.37\%) AML-patients. Among the non-Aspergillus moulds, mucormycosis was the most prevalent, occurring in 24 (2.56\%) HM-patients, with the ALL-subgroup most commonly affected $(\mathrm{N}=5$; $0.75 \%$ ). IC was most frequently identified in allo-HSCT-recipients $(\mathrm{N}=8 ; 2.38 \%)$ and ALL $(\mathrm{N}=15$; $2.26 \%)$ patients.
For HM-patients, intensive care unit (ICU) admission was higher in IFD-cases $(5.53 \%)$ than non-IFD controls $(1.82 \% ; \mathrm{p}<0.001)$. Median length of stay in ICU was approximately 2.5 -times greater in IFD-cases (6.08-days) compared to uninfected-controls (2.46-days; $\mathrm{p}<0.001)$. The median duration of mechanical ventilation was 3.5-times greater in IFD-cases versus non-IFD controls (6.83-days versus 1.96-days; $\mathrm{p}<0.001)$. A significantly 
Table 2 Invasive Fungal Disease Incidence Stratified by Haematological Malignancy and Haematopoietic Stem Cell Transplantation from Index Hospitalisation, 2005 - 2016

\begin{tabular}{ll}
\hline Haematological malignancy and haematopoietic stem cell transplantation & Invasive fungal disease; incidence (\%) \\
\hline Allogeneic-haematopoietic stem cell transplantation $(\mathrm{N}=335)$ & $41(12.2)$ \\
Acute lymphoblastic leukaemia $(\mathrm{N}=664)$ & $75(11)$ \\
Acute myeloid leukaemia $(\mathrm{N}=2,644)$ & $249(9.42)$ \\
Autologous-haematopoietic stem cell transplantation $(\mathrm{N}=1,449)$ & $70(4.83)$ \\
Aplastic anaemia $(\mathrm{N}=7,121)$ & $101(1.42)$ \\
Chronic lymphocytic leukaemia $(\mathrm{N}=3,459)$ & $46(1.33)$ \\
Non-Hodgkin lymphoma $(\mathrm{N}=15,267)$ & $192(1.26)$ \\
Multiple myeloma $(\mathrm{N}=5,614)$ & $58(1.03)$ \\
Hodgkin lymphoma $(\mathrm{N}=2,030)$ & $17(0.84)$ \\
Chronic myeloid leukaemia $(\mathrm{N}=1,240)$ & $10(0.81)$ \\
Other haematological malignancies $(\mathrm{N}=1,897)^{a}$ & $22(1.16)$ \\
\hline
\end{tabular}

${ }^{\mathrm{a}}$ Myelodysplastic syndrome patients included in 'Other' due to a small patient cohort.

greater proportion of IFD-cases required haemodialysis than controls among HM-patients (10\% versus $1.49 \%$; $\mathrm{p}<0.001)$ and HSCT-recipients $(9.01 \%$ versus $3.75 \%$; $\mathrm{p}=0.007)$, respectively. One-, three-, six- and twelve-month mortality from time of HM-diagnosis was consistently higher in IFD-cases in both HM and HSCT populations compared to controls (Table 1).

\section{Time to invasive fungal disease}

The median time to IFD-onset was shortest among AML-patients (3-months) and longest for multiple myeloma (MM) patients (22-months) (Fig. 3). Of the
13 allo-HSCT-recipients with graft-versus-host-disease (GVHD), the median time to IFD was markedly shorter (1-month) compared to 91 allo-HSCT-recipients without GVHD who developed IFD (6-months) (Additional file 5).

\section{Survival analysis and risk of mortality}

The hazard ratio for IFD-onset post-HM-diagnosis was 1.24 (95\% CI: 0.88-1.93; $\mathrm{p}=0.329$ ) denoting that the instantaneous risk of mortality was 1.24-times greater when a HM-patient developed an IFD within twelve-months from index hospitalisation. After stratifying by IFD, the shortest median survival time was for mucormycosis-

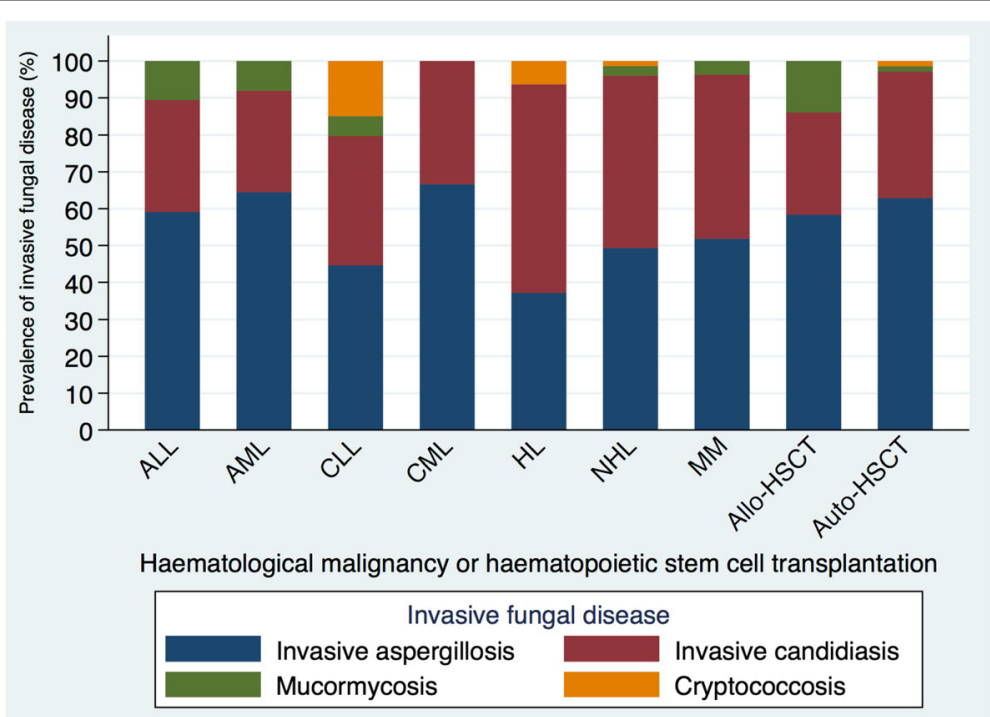

Fig. 2 Prevalence of invasive fungal disease among cases by haematological malignancy or haematopoietic stem cell transplantation. ALL, acute lymphoblastic leukaemia; allo-HSCT, allogeneic haematopoietic stem cell transplantation; AML, acute myeloid leukaemia; auto-HSCT, autologous haematopoietic stem cell transplantation; CLL, chronic lymphocytic leukaemia; CML, chronic myeloid leukaemia; HL, Hodgkin-lymphoma; MM, multiple myeloma; NHL, non-Hodgkin lymphoma. 
infected patients (median survival time: 3-months from IFD diagnosis), followed by IA and IC (7-months each) (Fig. 4).

\section{Time-series analysis of the risk-adjusted incidence of invasive fungal disease}

Over the 11-year study period, the incidence of IFD per 1,000 bed-days decreased by $0.28 \%$ (Fig. 5). A seasonal trend in IFD incidence was evident with peaks coinciding with the onset of spring (September to November in Australia).

\section{Risk factors for invasive fungal disease}

A multivariate analysis assessing risk factors for IFD identified 10 significant covariates, including neutropenia, acute renal failure, ICU admission, residence in rural Victoria, haemodialysis, viral infection, Clostridium difficile infection, haematological malignancy, increasing age in years and admission to a metropolitan hospital (Table 3).

\section{Discussion}

This is the first comprehensive study of IFD incidence and survival in Victoria among haematology patients over a period of 11 years and highlights the possibilities of data linkage, but also the shortcomings of administrative data for surveillance of a rare disease. The most striking finding from this study is the low overall incidence of IFD among HM-patients (2.04\%) and HSCT-recipients (6.29\%). It is likely that IFD is under-reported at a hospital level in coding data $[14,15]$ and this translates into the data generated by the VAED. Despite this shortcoming, we were able to identify periods of high-risk for a range of HMs, seasonal trends in IFD and an overall decrease in IFD incidence over the 11 years. In addition, access to a high number of clinical covariates allowed for exploration of risk-factors for IFD through multivariate regression analysis that may assist in tailoring preventative therapies like antifungal prophylaxis according to individual risk.

The epidemiological trends in IFD incidence and mortality in the HM population has historically been limited to institutional-specific reports and multicentre studies focusing predominantly on IA, IC and mucormycosis $[1,4,16]$. By contrast, through data linkage of hospital administrative data (VAED) with state-based registries (VCR and VDI), we described epidemiological trends among all HM-patients. Mould diseases predominated in keeping with global trends [6], accounting for $61 \%$ of IFD compared to $39 \%$ due to invasive candidiasis. Among mould diseases, IA was the predominant species (91\%), followed by mucormycosis (8.76\%); a finding concordant with recent studies [17, 18]. Mucormycosis most commonly affected allo-HSCT-recipients (1.19\%), followed by ALL (0.75\%) and AML (0.45\%) patients and was associated with the shortest median survival time of 3-months compared to 7-months each for IA and IC. The emergence of mucormycosis as the predominant non-Aspergillus mould is consistent with the largest

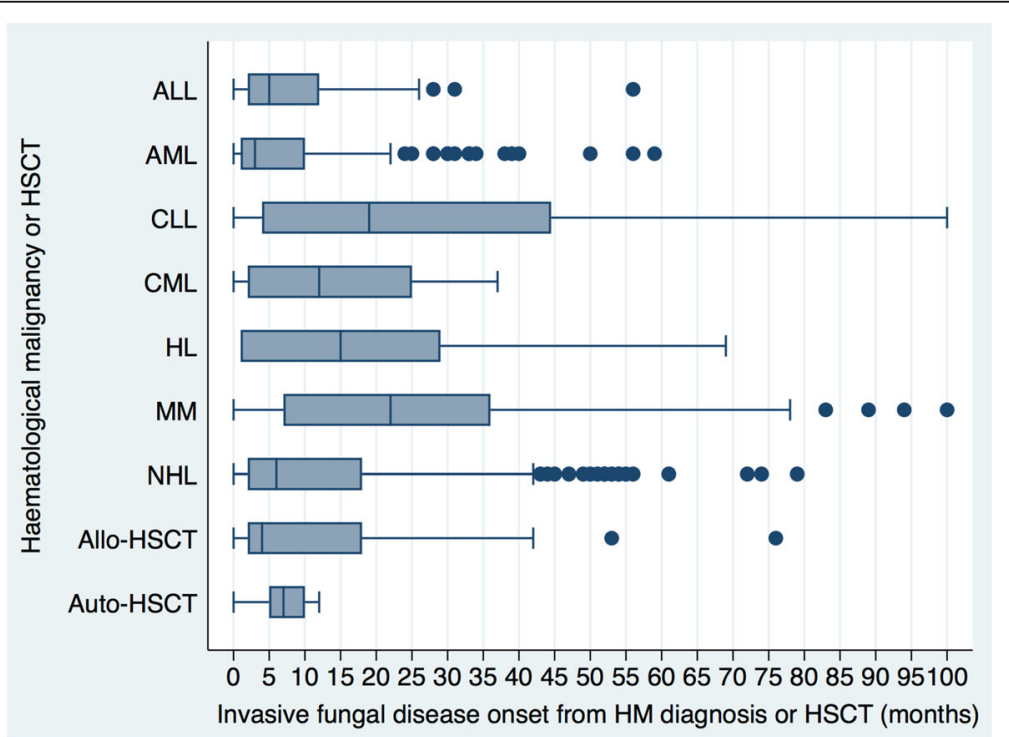

Fig. 3 Overall distribution of time (months) to invasive fungal disease stratified by haematological malignancy and haematopoietic stem cell transplantation. ALL, acute lymphoblastic leukaemia; allo-HSCT, allogeneic-haematopoietic stem cell transplantation; AML, acute myeloid leukaemia; auto-HSCT, autologous-haematopoietic stem cell transplantation; CLL, chronic lymphoblastic leukaemia; CML, chronic myeloid leukaemia; HL, Hodgkin lymphoma; HM, haematological malignancy; IFD, invasive fungal disease; IQR, inter-quartile range; MM, multiple myeloma; NHL, non-Hodgkin lymphoma. Median [IQR] time (months) to IFD: ALL, 5 [2 - 12]; AML, 3 [1 - 10]; CLL, 19 [4 - 45]; CML, 12 [2 - 25]; HL, 15 [1 - 29]; MM, 22 [7 - 36]; NHL, 6 [2 -18]; allogeneic-HSCT, 4 [2 - 18]; autologous-HSCT, 7 [5 - 10] 


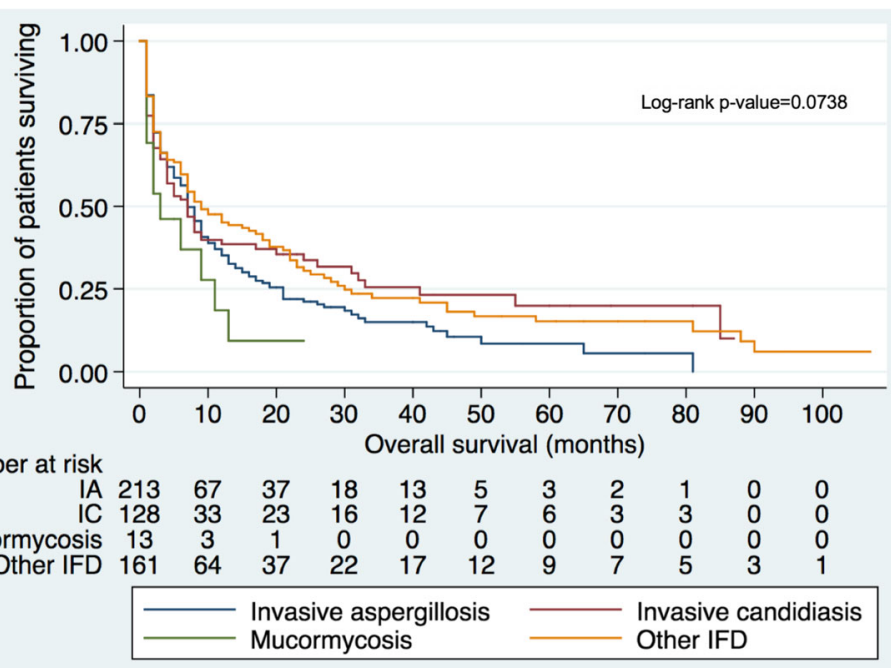

Fig. 4 Kaplan-Meier survival curves illustrating overall survival (months) from invasive fungal disease diagnosis between invasive aspergillosis (IA), invasive candidiasis (IC), mucormycosis and other invasive fungal disease. IFD, invasive fungal disease.

multicentre surveillance study of IFD epidemiology in HSCT recipients [6] and is likely due to several factors including longer survival post-HSCT [6, 19].

A higher IFD incidence (11\%) in ALL compared to AML (9.42\%) is intriguing but confirms that the ALL cohort is an emerging subgroup at high-risk of IFD with a variable fungal incidence ranging from $6.5-12 \%[20,21]$. Prophylaxis with azole antifungals is contra-indicated due to the drug-drug interactions with vinca alkaloids used in ALL treatment regimens [22]; but the lack of an approved standard of care from clinical trial data [21] means that clinical variation in prophylactic strategies for ALL patients is likely [23]. Patients with CLL have the third-highest IFD incidence (1.33\%) and are increasingly recognised as being at high-risk of IFD due to a shift from chemo-immunotherapies to agents targeting specified B-lymphocyte pathways [24]. Indeed, IFD incidence in non-Hodgkin lymphoma (NHL) (1.26\%) was the fourth highest of all HM (Table 2) which may reflect the effects of multi-agent chemotherapy in combination with immunotherapy used to treat NHL [25].

Attempts at clinical risk-stratification for IFD have been crude and restricted to broadly identifying low-, intermediate- and high-risk groups [20] in a large part

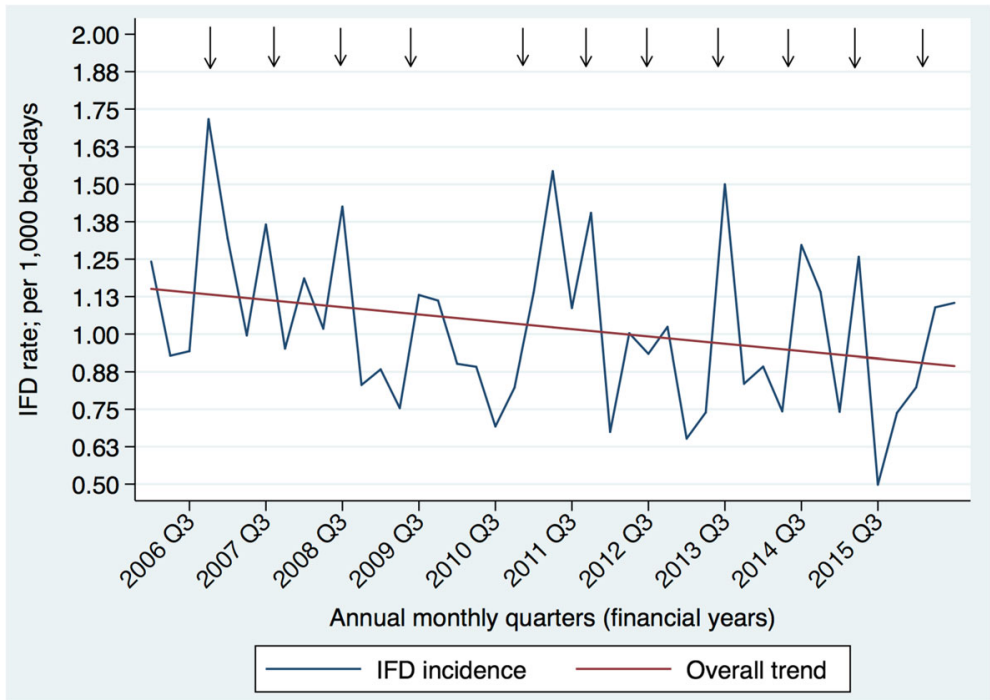

Fig. 5 The quarterly incidence (financial years; \%) of invasive fungal disease (IFD) in Victoria (2005 - 2016) along with a linear fitted values line. Black arrows indicate the spring months. Q, quarter 
Table 3 Multivariate Logistic Regression Analysis of Statistically Significant Risk Factors for Invasive Fungal Diseases among Haematological Malignancy Patients Twelve-Months from Induction Chemotherapy, 2005 - 2016

\begin{tabular}{|c|c|c|c|c|}
\hline Covariate & Odds ratio (OR) & Coefficient & 95\% confidence interval OR & $p$-value \\
\hline Acute renal failure & 1.895 & 0.639 & $1.284-2.798$ & $<0.001$ \\
\hline \multicolumn{5}{|l|}{ Age (ten years) ${ }^{a}$} \\
\hline $25-34$ & 1.030 & 0.029 & $0.555-1.910$ & 0.926 \\
\hline $35-44$ & 0.996 & -0.004 & $0.554-1.790$ & 0.988 \\
\hline $45-54$ & 1.537 & 0.430 & $0.916-2.580$ & 0.103 \\
\hline $55-64$ & 1.345 & 0.296 & $0.813-2.225$ & 0.249 \\
\hline $65-74$ & 1.134 & 0.126 & $0.690-1.864$ & 0.619 \\
\hline $75-84$ & 0.805 & -0.217 & $0.482-1.344$ & 0.406 \\
\hline $85-94$ & 0.355 & -1.037 & $0.186-0.678$ & 0.002 \\
\hline $95+$ & 0.339 & -1.083 & $0.045-2.575$ & 0.295 \\
\hline Clostridium difficile infection & 2.792 & 1.027 & $1.203-6.480$ & 0.017 \\
\hline \multicolumn{5}{|l|}{ Haematological malignancy ${ }^{a}$} \\
\hline ALL & 11.400 & 2.434 & $5.215-24.918$ & $<0.001$ \\
\hline AML & 8.743 & 2.168 & $4.247-17.998$ & $<0.001$ \\
\hline$C L L$ & 1.734 & 0.550 & $0.793-3.790$ & 0.168 \\
\hline $\mathrm{HL}$ & 0.806 & -0.216 & $0.326-1.991$ & 0.640 \\
\hline MDS & 1.194 & 0.177 & $0.133-10.708$ & 0.874 \\
\hline $\mathrm{NHL}$ & 1.503 & 0.408 & $0.728-3.103$ & 0.270 \\
\hline MM & 1.221 & 0.199 & $0.570-2.614$ & 0.608 \\
\hline Haemodialysis & 3.220 & 1.169 & $1.380-7.510$ & 0.007 \\
\hline Admission to a rural hospital ${ }^{a}$ & 0.508 & -0.676 & $0.368-0.702$ & $<0.001$ \\
\hline Intensive care unit admission & 1.626 & 0.486 & $1.324-1.997$ & $<0.001$ \\
\hline Neutropenia & 2.176 & 0.778 & $1.703-2.781$ & $<0.001$ \\
\hline \multicolumn{5}{|l|}{ Region of residence ${ }^{a}$} \\
\hline Metropolitan Victoria & 0.762 & -0.271 & $0.596-0.976$ & 0.031 \\
\hline Interstate & 0.482 & -0.730 & $0.273-0.851$ & 0.012 \\
\hline Unknown & 0.941 & -0.061 & $0.278-3.181$ & 0.922 \\
\hline Viral infection & 3.117 & 1.137 & $1.081-8.987$ & 0.035 \\
\hline CONSTANT & 0.015 & -4.175 & $-5.146--3.204$ & $<0.001$ \\
\hline
\end{tabular}

${ }^{a}$ Reference categories for polychotomous independent variables: Age (ten years), 15 - 24; haematological malignancy, CML; hospital region, metropolitan hospital; region of residence, rural Victoria. Reference categories were chosen as the lowest risk-burden for invasive fungal disease

Abbreviations: $A L L$ acute lymphoblastic leukaemia, $A M L$ acute myeloblastic leukaemia, CLL chronic lymphoblastic leukaemia, $H L$ Hodgkin-lymphoma, MDS myelodysplastic syndrome, MM multiple myeloma, $N H L$ non-Hodgkin lymphoma, OR odds ratio

because large datasets for a rare disease like IFD do not exist [26]. We confirmed risk-factors that are associated with IFD including viral infections [27], admission to a rural hospital that may reflect rural place of residence [20] and Clostridium difficile infection $(\mathrm{p}<0.05)$ which has not been previously described as a risk-factor for IFD but is prevalent in immunocompromised populations [28]. In addition, access to a high number of clinical covariates allowed exploration of a predictive tool to quantify IFD-risk at the patient-level informed by a range of risk-factors elucidated on multivariate regression analysis. We identified periods of high-risk for IFD from the time of HM diagnosis with the shortest median time seen in AML-patients (3-months) and the longest in patients with MM (22-months). The latter finding reflects the cumulative immunosuppression associated with successive lines of therapy, including immunomodulatory chemotherapies and prolonged corticosteroid exposure that is characteristic of myeloma treatment [29]. Consistent with intervals of high-risk described in Hammond et al. [30], risk-periods of IFD for other HMs were defined including ALL (5-months) and NHL (6-months) [20]. The shorter median time to IFD-onset after transplantation among GVHD-positive- (1-month) compared to 
GVHD-negative-HSCT-recipients (6-months) reflects the increased immunosuppression associated with GVHD and its treatment [31] (Additional file 5).

During the study period, there was an overall decreasing trend in IFD incidence in Victoria. The $0.28 \%$ decline in IFD incidence from 2005-2016, is contrary to the overall $3.5 \%$ increase observed in an earlier retrospective study from 1995-1999 [4]. This progressive decrease in IFD incidence is likely multifactorial and related to improved supportive care encompassing broad-spectrum antifungal prophylactic regimens for some subgroup of patients (e.g. AML, HSCT-recipients with GVHD), coupled with improved diagnostic investigations [32], clinical guidelines for IFD [33], better management of GVHD [34], cytomegalovirus prevention [27] and the introduction of high-efficiency particulate air filtration systems into some transplantation wards [35]. While an intensive diagnostic approach incorporating non-culture-based tests increases diagnostic yield [36] and corresponding fungal incidence, their availability is limited with only $35 \%$ of centres in a national Australian survey providing on-site Aspergillus galactomannan (GM) or polymerase chain reaction (PCR) diagnostic tests. Therefore, it seems likely that the decline in IFD incidence we observed may be explained by the uptake of mould-active prophylaxis targeting high-risk groups, as seen in a major Victorian transplant centre, which reported a reduction in IFD incidence in patients with AML from $25 \%$ with fluconazole use to $3 \%$ with posaconazole use over a 12-year period [37]. Indeed, this practice is widespread, with a nationwide survey reporting that posaconazole prophylaxis was used in $90 \%$ of AML patients undergoing chemotherapy and $68 \%$ of allogeneic-HSCT recipients, with lower rates among ALL patients of 53\% [38], highlighting the lack of a standardised approach in this patient group. Consistent with the $5.7 \%$ increase in IA incidence during the warmer months as reported by Panackal et al. [39], the peaks in IFD incidence at the onset of spring indicates seasonality not previously described in the southern hemisphere (Fig. 5). This knowledge could ensure that preventative strategies, coupled with enhanced surveillance, also take seasonality into consideration.

Linkage of administrative and clinical datasets could potentially improve knowledge discovery for a rare disease such as IFD, but is contingent on the completeness of hospital-level data collection. Cancer surveillance systems that leverage data linkage between the VCR and clinical registries is considered a technological solution to more accurately determine the epidemiology of rare leukaemia in Victoria [40]. Limited international [14] and Australian data [15] suggest that IFD are under-reported in hospital administrative systems. This is in a large part because fungal surveillance is difficult requiring multidisciplinary input followed by adjudication of cases according to complex definitions [41]. Chang et al. described the poor sensitivity of coding data of $32 \%$ for proven/probable IA in HSCT-recipients and its poor positive predictive value of $15 \%$ [14]. However, the quality of coding practice is dependent on the quality of medical record documentation, particularly discharge summaries and this has been shown to be suboptimal for IFD even when fungaemia was present [15]. Institutional underreporting has implications for hospital reimbursement but also diminishes the utility of large datasets for rare disease surveillance. Furthermore, the fact that no HSCT-recipient with an IFD was admitted to the ICU in our study is implausible considering that patients with mucormycosis frequently have multiple surgeries and require ICU support (Table 1) [6]. The introduction of sensitive machine learning-based data analytics [42] could enable real-time surveillance of IFD and improve the quality of fungal reporting at the hospital level where most of these infections are managed.

There are several limitations to this study. The quality of coding data for IFD is the foremost consideration as previously discussed. Linkage of the VAED with the VCR was only available between the $1^{\text {st }}$ January 2008 and the $31^{\text {st }}$ December 2014. Thus, we relied on the VAED to identify index hospitalisations for the other years without verification against confirmed HM-diagnosis from the VCR. Secondly, as a retrospective study, our analysis is subject to misclassification or miscoding of IFD [14]. Finally, the risk-factors we identified from multivariate analysis require validation against a separate dataset, but large datasets for IFD are currently unavailable due to the lack of comprehensive surveillance systems.

\section{Conclusions}

The true burden of IFD among haematology-oncology patients is difficult to accurately determine from hospitalbased data. We hypothesise that the true incidence is likely to be higher but without implementation of surveillance systems, it will remain underestimated. The migration of hospital systems both within Victoria, and globally, to the electronic medical record provides an opportunity to improve IFD surveillance through innovative data mining techniques [42].

\section{Additional files}

Additional file 1: STROBE Checklist. STROBE Statement and checklist of items that should be included in reports of cohort studies. (DOC $87 \mathrm{~kb}$ )

Additional file 2: Inclusion ICD-10-AM Codes and Invasive Fungal Disease Diagnoses Stratified by Haematological Malignancy and Haematopoietic Stem Cell Transplantation from Index Hospitalisation, 2005 - 2016. Administrative coding data used to identify invasive fungal diseases (IFD) and haematological malignancy and haemopoietic stem cell transplantation (HSCT) recipients that were included in the study. Additional file 2 also contains data on the incidence of individual IFD diagnoses stratified by underlying malignancy and HSCT. (DOCX 54 kb) 
Additional file 3: : Exclusion ICD-10-AM Codes. Administrative coding data used to identify superficial fungal infections that were excluded from this study. (DOCX $14 \mathrm{~kb}$ )

Additional file 4: Receiver Operating Curve and C-Statistic. Receiver operating curve and its corresponding C-statistic for the evaluation of the multivariable logistic regression model. (DOCX $162 \mathrm{~kb}$ )

Additional file 5: Distribution of time (months) to invasive fungal disease among allogeneic haematopoietic stem cell transplantation (HSCT) recipients stratified by graft-versus-host disease (GVHD) status post-transplantation (GVHD negative, $\mathrm{N}=13$; $\mathrm{GVHD}$ positive, $\mathrm{N}=28$ ). Boxplot stratified by allogeneic-HSCT recipients with and without GVHD detailing the median time (in months) to invasive fungal disease onset. (DOCX $184 \mathrm{~kb})$

\section{Abbreviations}

ALL: Acute lymphoblastic leukaemia; Allo: Allogeneic; AML: Acute myeloid leukaemia; Auto: Autologous; GM: Galactomannan; GVHD: Graft-versus-hostdisease; HM: Haematological malignancy; HSCT: Haematopoietic stem cell transplantation; IA: Invasive aspergillosis; IC: Invasive candidiasis; ICD-10AM: International Statistical Classification of Diseases and Related Health Problems, Tenth Revision, Australian Modification; ICU: Intensive care unit; IFD: Invasive fungal disease; MM: Multiple myeloma; NHL: Non-Hodgkin lymphoma; PCR: Polymerase chain reaction; STROBE: STrengthening the Reporting of OBservational studies in Epidemiology; VAED: Victorian Admitted Episodes Dataset; VCR: Victorian Cancer Registry; VDI: Victorian Death Index; VDLU: Victorian Data Linkages Unit

\section{Acknowledgements}

The authors would like to acknowledge the State Government of Victoria's Department of Health and Human Services, the data custodians of the (Victorian Admitted Episodes Dataset) VAED, the (Victorian Cancer Registry) VCR and (Victorian Death Index) VDI for allowing the investigators to access their data. We also thank Matthew Ha and associates of the Victorian Government's Systems Intelligence and Analytics Branch and the (Victorian Data Linkages Unit) VDLU for linking our data between major Victorian data consortiums. We wish to thank Tracy Burgess and colleagues from Alfred Health's Health Information Services for allowing access to the (International Statistical Classification of Diseases and Related Health Problems, Tenth Revision Australian Modification) ICD-10-AM coding manuals. The authors thank Fiona Miles from the Monash University Advisory Board and Tsharni Zazryn from the Monash Research Office for authorising administrative access to the merged VAED dataset. A.Y.P. acknowledges support from the Australian National Health and Medical Research Council through a Practitioner Fellowship.

\section{Funding}

This was an unfunded study.

\section{Availability of data and materials}

The datasets generated and/or analysed during the current study are not publicly available due to ethics restrictions (i.e. placing the dataset in a publicly available repository has not been approved by the relevant ethics committee because it contains highly sensitive information) but are available from the corresponding author on reasonable request and pending approval of the relevant ethics committee.

\section{Previous presentations}

This research was presented in part at the 2018 Australasian Society for Infectious Diseases Annual Scientific Meeting at the Gold Coast, Queensland, Australia $10^{\text {th }}-12^{\text {th }}$ May 2018.

\section{Authors' contributions}

Manuscript preparation and statistical analysis was by J.C.V., C.O.M. and M.R.A.-R.; study conception and design were by M.R.A.-R.; data retrieval was by J.C.V., C.O.M. and M.R.A.-R.; statistical support was provided from M.A.T.; J.C.V., C.O.M., M.A.T., D.L., S.P., A.Y.P. and M.R.A.-R. contributed to the data interpretation and critical revision of the manuscript. All authors have read and approved the manuscript.

\section{Authors' information}

J.C.V. BBiomedSc(Hons), Ph.D. Candidate and Research Fellow, University of Melbourne, the Peter MacCallum Cancer Centre and the National Centre for Infections in Cancer; C.O.M. MB, BCh, FRACP, Grad Dip Clin Epi, Ph.D., Infectious Diseases Physician, Monash University and Alfred Health; M.A.T. MBiostat, BSC., Research Fellow and Biostatistician, Austin Health; D.L. MBBS (Hons), BMedSC, FRACP, Ph.D., CertHealthEcon, Professor of Clinical Outcomes Research; S.P. MBBS; FRACP, FRCPA, Haematologist, Alfred Health; A.Y.P. MBBS Ph.D. MPH FRACP, Infectious Diseases Physician, Monash University and Alfred Health; M.R.A.-R. MBBS (Hons) FRACP Ph.D., Infectious Diseases Physician, Monash University and Alfred Health.

\section{Ethics approval and consent to participate}

Written ethics approval was granted by the Alfred Health Human Research Ethics Committee (project number: 93/17). The need for informed consent was deemed unnecessary according to national regulations in accordance with the National Health and Medical Research Council National Statement on Ethical Conduct in Human Research (2007 and updates) [43]. Administrative permission to access the raw data from the Victorian Data Linkages Unit was granted from Ms. Fiona Miles of the Monash University Advisory Board and Dr. Tsharni Zazryn of the Monash Research Office.

\section{Consent for publication}

Not applicable.

\section{Competing interests}

The authors declare that they have no competing interests.

\section{Publisher's Note}

Springer Nature remains neutral with regard to jurisdictional claims in published maps and institutional affiliations.

\section{Author details}

'Department of Infectious Diseases, Alfred Health and Central Clinical School, Monash University, Melbourne, Victoria, Australia. ${ }^{2}$ Department of Epidemiology and Preventive Medicine, Monash University, Melbourne, Victoria, Australia. ${ }^{3}$ Malignant Haematology and Stem Cell Transplantation Service, Alfred Health, Melbourne, Victoria, Australia. ${ }^{4}$ Infection and Immunity Program, Monash Biomedicine Discovery Institute, Department of Microbiology, Monash University, Clayton, Victoria, Australia. ${ }^{5}$ General Medicine Unit, Alfred Health, Melbourne, Victoria, Australia. ${ }^{6}$ Cancer Research Division, Level 13, Peter MacCallum Cancer Centre, Victorian Comprehensive Cancer Centre, 305 Grattan Street, Melbourne, Victoria 3000, Australia.

Received: 20 September 2018 Accepted: 13 March 2019 Published online: 21 March 2019

\section{References}

1. Slavin M, van Hal S, Sorrell TC, Lee A, Marriott DJ, Daveson K, Kennedy K, Hajkowicz K, Halliday C, Athan E, et al. Invasive infections due to filamentous fungi other than Aspergillus: Epidemiology and determinants of mortality. Clin Microbiol Infect. 2015;21:490 e491-10.

2. Nivoix Y, Velten M, Letscher-Bru V, Moghaddam A, Natarajan-Ame S, Fohrer C, Lioure B, Bilger K, Lutun P, Marcellin L, et al. Factors associated with overall and attributable mortality in invasive aspergillosis. Clin Infect Dis. 2008:47:1176-84.

3. Even C, Bastuji-Garin S, Hicheri Y, Pautas C, Botterel F, Maury S, Cabanne L, Bretagne S, Cordonnier C. Impact of invasive fungal disease on the chemotherapy schedule and event-free survival in acute leukemia patients who survived fungal disease: A case-control study. Haematologica. 2011;96:337-41.

4. Slavin M, Fastenau J, Sukarom I, Mavros P, Crowley S, Gerth WC. Burden of hospitalization of patients with Candida and Aspergillus infections in Australia. J Infect Dis Med. 2004;8:111-20.

5. Lingaratnam S, Thursky KA, Slavin MA, Kirsa SW, Bennett CA, Worth LJ. The disease and economic burden of neutropenic fever in adult patients in Australian cancer treatment centres 2008: Analysis of the Victorian Admitted Episodes Dataset. Intern Med J. 2011:41:121-9.

6. Kontoyiannis DP, Marr KA, Park BJ, Alexander BD, Anaissie EJ, Walsh TJ, Ito J, Andes DR, Baddley JW, Brown JM, et al. Prospective surveillance for invasive fungal infections in hematopoietic stem cell transplant recipients, 2001- 
2006: Overview of the Transplant-Associated Infection Surveillance Network (TRANSNET) Database. Clin Infect Dis. 2010;50:1091-100.

7. Australian Bureau of Statistics: Australian Demographic Statistics [http://www. abs.gov.au/ausstats/abs@.nsf/0/D56C4A3E41586764CA2581A70015893E? Opendocument]. Accessed 16 Aug 2018.

8. Gooley TA, Chien JW, Pergam SA, Hingorani S, Sorror ML, Boeckh M, Martin PJ, Sandmaier BM, Marr KA, Appelbaum FR, et al. Reduced mortality after allogeneic hematopoietic-cell transplantation. N Engl J Med. 2010;363:2091-101.

9. Department of Health and Human Services, State Government of Victoria. Victorian Admitted Episodes Dataset (VAED) manual. 25 edition. Melbourne: State Government of Victoria; 2015-2016:1-29.

10. McQuilten ZK, Wood EM, Polizzotto MN, Campbell $\amalg$, Wall M, Curtis DJ, Farrugia H, McNeil JJ, Sundararajan V. Underestimation of myelodysplastic syndrome incidence by cancer registries: Results from a population-based data linkage study. Cancer. 2014;120:1686-94.

11. PLOS Medicine Editors. Observational studies: getting clear about transparency. PLoS Med. 2014;11:e1001711.

12. Muller C, MacLehose RF. Estimating predicted probabilities from logistic regression: Different methods correspond to different target populations. Int J Epidemiol. 2014;43:962-70.

13. Menendez ME, Janssen SJ, Ring D. Electronic health record-based triggers to detect adverse events after outpatient orthopaedic surgery. BMJ Qual Saf. 2016;25:25-30.

14. Chang DC, Burwell LA, Lyon GM, Pappas PG, Chiller TM, Wannemuehler KA, Fridkin SK, Park BJ. Comparison of the use of administrative data and an active system for surveillance of invasive aspergillosis. Infect Control Hosp Epidemiol. 2008;29:25-30.

15. Nguyen Q, Reid B. A comparison of two sources of data on fungaemia in two hospitals. Health Inf Manag. 2005;34:88-93.

16. Liu YC, Chien SH, Fan NW, Hu MH, Gau JP, Liu CJ, Yu YB, Liu CY, Hsiao LT, Liu JH, et al. Incidence and risk factors of probable and proven invasive fungal infection in adult patients receiving allogeneic hematopoietic stem cell transplantation. J Microbiol Immunol Infect. 2016;49:567-74.

17. Vallabhaneni S, Benedict K, Derado G, Mody RK. Trends in hospitlizations related to invasive aspergillosis and mucormycosis in the United States, 2000 - 2013. Open Forum Infect Dis. 2017:4:1-8.

18. Zilberberg MD, Nathanson BH, Harrington $R$, Spalding JR, Shorr AF. Epidemiology and outcomes of hospitalizations with invasive aspergillosis in the United States, 2009-2013. Clin Infect Dis. 2018. https://doi.org/10.1093/cid/ciy181.

19. Lanternier F, Sun H-Y, Ribaud P, Singh N, Kontoyiannis DP, Lortholary O. Mucormycosis in organ and stem cell transplant recipients. Clin Infect Dis. 2012;54:1629-36.

20. Pagano L, Busca A, Candoni A, Cattaneo C, Cesaro S, Fanci R, Nadali G, Potenza L, Russo D, Tumbarello M, et al. Risk stratification for invasive fungal infections in patients with hematological malignancies: SEIFEM recommendations. Blood Rev. 2017;31:17-29.

21. Cornely OA, Leguay T, Maertens J, Vehreschild MJGT, Anagnostopoulos A, Costagnola C, Verga L, Rieger C, Kondakci M, Härter G, et al. Randomized comparison of liposomal amphotericin B versus placebo to prevent invasive mycoses in acute lymphoblastic leukaemia. J Antimicrob Chemother. 2017:72:2359-67.

22. Bassan R, Hölzer D. Modern therapy of acute lymphoblastic leukemia. J Clin Oncol. 2010:29:532-43.

23. Nicolato A, Nouér SA, Garnica M, Portugal R, Maiolino A, Nucci M. Invasive fungal diseases in patients with acute lymphoid leukemia. Leuk Lymphoma. 2016;57:2084-9.

24. Teh BW, Tam CS, Handunnetti S, Worth LJ, Slavin MA. Infections in patients with chronic lymphocytic leukaemia: Mitigating risk in the era of targeted therapies. Blood Rev. 2018;32(6):499-507.

25. Chamilos G, Lionakis MS, Kontoyiannis DP. Call for action: Invasive fungal infections associated with ibrutinib and other small molecule kinase inhibitors targeting immune signaling pathways. Clin Infect Dis. 2018;66:140-8.

26. Stanzani $M$, Lewis RE, Fiacchini M, Ricci $P$, Tumietto F, Viale $P$, Ambretti S, Baccarani M, Cavo M, Vianelli N. A risk prediction score for invasive mold disease in patients with hematological malignancies. PLoS One. 2013;8:e75531.

27. Yong MK, Ananda-Rajah MR, Cameron PU, Morrissey CO, Spencer A, Ritchie $D$, Cheng AC, Lewin SR, Slavin M. Cytomegalovirus reactivation is associated with increased risk of late-onset invasive fungal disease after allogeneic hematopoietic stem cell transplantation: A multicenter study in the current era of viral load monitoring. Biol Blood Marrow Transplant. 2017;23:1961-7.

28. Cannon CM, Musuuza JS, Barker AK, Duster M, Juckett MB, Pop-Vicas AE, Sadfar N. Risk of Clostridium difficile infection in hematology-oncology patients colonized with toxigenic C. difficile. Infect Control Hosp Epidemiol. 2017;38:718-20.

29. Teh BW, Harrison SJ, Worth LJ, Thursky KA, Slavin MA. Infection risk with immunomodulatory and proteasome inhibitor-based therapies across treatment phases for multiple myeloma: A systematic review and metaanalysis. Eur J Cancer. 2016;67:21-37.

30. Hammond SP, Marty FM, Bryar JM, DeAngelo DJ, Baden LR. Invasive fungal disease in patients treated for newly diagnosed acute leukemia. Am J Hematol. 2010;85:695-9.

31. Miller HK, Braun TM, Stillwell T, Harris AC, Choi S, Connelly J, Couriel D, Goldstein S, Kitko CL, Magenau J, et al. Infectious risk after allogeneic hematopoietic cell transplantation complicated by acute graft-versus-host disease. Biol Blood Marrow Transplant. 2017;23:522-8.

32. Morrissey CO, Gilroy NM, Macesic N, Walker P, Ananda-Rajah M, May M, Heath $\mathrm{CH}$, Grigg A, Bardy PG, Kwan J, et al. Consensus guidelines for the use of empiric and diagnostic-driven antifungal treatment strategies in haematological malignancy, 2014. Intern Med J. 2014;44:1298-314.

33. Slavin MA, Thursky KA, Worth LJ, Chang CC, Morrissey CO, Blyth CC, Chen SC, Szer J. Introduction to the updated Australian and New Zealand consensus guidelines for the use of antifungal agents in the haematology/ oncology setting, 2014. Intern Med J. 2014;44:1267-76.

34. Ferrara JLM. Advances in the clinical management of GVHD. Best Pract Res Clin Haematol. 2008;21:677-82.

35. Eckmanns $T$, Rüden $H$, Gastmeier $P$. The influence of high-efficiency particulate air filtration on mortality and fungal infection among highly immunosuppressed patients: A systematic review. J Infect Dis. 2006;193:1408-18.

36. Ceesay MM, Desai SR, Berry L, Cleverley J, Kibbler CC, Pomplun S, Nicholson AG, Douiri A, Wade J, Smith M, et al. A comprehensive diagnostic approach using galactomannan, targeted $\beta$-D-glucan, baseline computerized tomography and biopsy yields a significant burden of invasive fungal disease in at risk haematology patients. Br J Haematol. 2015;168:219-29.

37. Ananda-Rajah MR, Grigg A, Downey MT, Bajel A, Spelman T, Cheng A, Thursky KT, Vincent J, Slavin MA. Comparative clinical effectiveness of prophylactic voriconazole/posaconazole to fluconazole/itraconazole in patients with acute myeloid leukemia/myelodysplastic syndrome undergoing cytotoxic chemotherapy over a 12-year period. Haematologica. 2012:97:459-63.

38. van Hal SJ, Gilroy NM, Morrissey CO, Worth LJ, Szer J, Tam CS, Chen SC, Thursky KA, Slavin MA. Survey of antifugal prophylaxis and fungal diagnostic tests employed in malignant haematology and haematopoietic stem cell transplantation (HSCT) in Australia and New Zealand. Intern Med J. 2014;44:1277-82.

39. Panackal AA, Li H, Kontoyiannis DP, Mori M, Perego CA, Boeckh M, Marr KA. Geoclimatic influences on invasive aspergillosis after hematopoietic stem cell transplantation. Clin Infect Dis. 2010;50:1588-97.

40. Ong DM, Farrugia H, Wei A. Therapy-related acute myeloid leukaemia and myelodysplastic syndrome in Victoria, Australia 2003-2014. Intern Med J. 2018:48:822-9.

41. De Pauw B, Walsh TJ, Donnelly JP, Stevens DA, Edwards JE, Calandra T, Pappas PG, Maertens J, Lortholary O, Kauffman CA, et al. Revised definitions of invasive fungal disease from the European Organization for Research and Treatment of Cancer/Invasive Fungal Infections Cooperative Group and the National Institute of Allergy and Infectious Diseases Mycoses Study Group (EORTC/MSG) Consensus Group. Clin Infect Dis. 2008:46:1813-21.

42. Ananda-Rajah MR, Bergmeir C, Petitjean F, Slavin MA, Thursky KA, Webb GI. Toward electronic surveillance of invasive mold diseases in hematologyoncology patients: An expert system combining natural language processing of chest computed tomography reports, microbiology, and antifungal drug data. Clin Cancer Inform. 2017. https://doi.org/10.1200/CCl.1217.00011.

43. The National Health and Medical Research Council, the Australian Research Council and the Australian Vice-Chancellors' Committee. National Statement on Ethical Conduct in Human Research 2007 (Updated May 2015). Canberra: National Health and Medical Research Council, Australian Research Council and the Australian Vice-Chancellor's Committee. Commonwealth of Australia; 2007. 